\title{
Updates in immunotherapy for hepatocellular carcinoma
}

\author{
Leonardo G. da Fonseca', Flair José Carrilho² \\ ${ }^{1}$ Medical Oncology, São Paulo Clínicas Liver Cancer Group, Hospital das Clínicas Complex, Instituto do Cancer do Estado de São \\ Paulo, University of São Paulo School of Medicine, São Paulo, SP 05403-000, Brazil. \\ ${ }^{2}$ Department of Gastroenterology, São Paulo Clínicas Liver Cancer Group, Hospital das Clínicas Complex, Instituto do Cancer do \\ Estado de São Paulo, University of São Paulo School of Medicine, São Paulo, SP 05403-000, Brazil.
}

Correspondence to: Dr. Leonardo G. da Fonseca, Medical Oncology, São Paulo Clínicas Liver Cancer Group, Hospital das Clínicas Complex, Instituto do Cancer do Estado de São Paulo, University of São Paulo School of Medicine, Av Dr. Eneás Carvalho de Aguiar, 255 ICHC - 9th floor, room 9159, São Paulo, SP 05403-000, Brazil. E-mail: I.fonseca@fm.usp.br

How to cite this article: da Fonseca LG, Carrilho FJ. Updates in immunotherapy for hepatocellular carcinoma. Hepatoma Res 2019;5:37. http://dx.doi.org/10.20517/2394-5079.2019.012

Received: 9 Sep 2019 First Decision: 29 Sep 2019 Revised: 7 Oct 2019 Accepted: 8 Oct 2019 Published: 13 Oct 2019

Science Editor: Dalbir Sandhu Copy Editor: Cai-Hong Wang Production Editor: Jing Yu

\begin{abstract}
Hepatocellular carcinoma (HCC) carries an unfavorable prognosis and novel therapeutic strategies are needed. Until now, only few systemic agents have improved survival in patients with advanced stage disease. Immunotherapy changed the landscape in several tumor types by producing unprecedented clinical outcomes with a favorable safety profile. Liver presents a particular immune-suppressive microenvironment and HCC develops in a background of chronic inflammation in the vast majority of cases. In this regard, immunotherapy may be a suitable strategy. Preliminary research focused on therapies involving immune cells and anti-tumor immune response for HCC has shown encouraging preliminary results. Immune checkpoint inhibitors, such the anti-PD-1/PD-L1 monoclonal antibodies, have provided durable responses in patients with advanced stage disease, although the pioneers phase III trials did not confirm survival superiority over the available agents. Cancer vaccines, adoptive cellular therapies and combinations of local modalities with immunotherapy are promising approaches under active research.
\end{abstract}

Keywords: Hepatocellular carcinoma, immunotherapy, immunosuppression, prognosis, immunology, antibody, vaccine 


\section{INTRODUCTION}

Hepatocellular carcinoma (HCC) is a leading cause of cancer-related morbimortality and represents a major health problem worldwide. HCC is ranked as the sixth most incident neoplasm and the fourth cause of cancer-related death ${ }^{[1]}$.

Remarkable progresses have been achieved in prevention, early detection and diagnosis. Concurrently, new therapeutic strategies have been developed both in localized and advanced stages, what is leading to a more favorable outcome for HCC patients comparing to the past decades. However, the field is continuously evolving, and important barriers need to be surpassed.

In a significant proportion of cases, HCC is diagnosed at later stages and more than half of the patients with localized disease will develop disease recurrence after locoregional treatments $\mathrm{s}^{[2]}$. At present, systemic treatment options are limited. Only a few drugs have showed survival improvement according to phase III trials. In 2008, sorafenib was the first drug that proved survival benefit according to the results of two pivotal trials ${ }^{[3,4]}$. A decade later, lenvatinib ${ }^{[5]}$ proved non-inferiority to sorafenib as a first-line option. In the second-line setting, regorafenib ${ }^{[6]}$, cabozantinib $^{[7]}$ and ramucirumab ${ }^{[8]}$ demonstrated positive results in placebo-controlled studies. Despite the incorporation of these new agents, the median survival of advanced HCC patients treated with systemic treatment still remains around 2 years ${ }^{[\rho]}$ and there exists an unmet need for innovative approaches.

One of the most notable advances in oncology over the last years is the use of immunotherapy, alongside with an increasing knowledge on how the immune system behaves during carcinogenesis and tumor progression. The idea to harness the immune system against cancer comes from the late 19th century. Based on observations that some patients presented tumor remission after developing erysipelas, William Coley proposed to inject a mixture of live and inactivated bacteria into in vivo tumors. Although there was not a clear explanation at that time, anti-tumor responses were achieved in different types of tumors with this intervention ${ }^{[10]}$. In 1957, Thomas and Burnet proposed the theory of immunosurveillance, in which lymphocytes played a role of sentinels to detect and destroy transformed cells ${ }^{[1]]}$. Afterwards, in the decade of 1970s, the use of tuberculosis vaccine with Bacille Calmette-Guérin was effective in preventing recurrence of urothelial carcinomas and is still applied nowadays ${ }^{[12]}$. In 1990s, interleukin (IL)-2 was approved for treatment of kidney cancer and melanoma. In the same decade, the first anti-CD20 monoclonal antibody, named rituximab, was approved for non-Hodgkin lymphoma ${ }^{[13]}$.

Currently, we are experiencing emerging trends in immunotherapy for treatment of several solid tumors, including HCC. The scope of this review is to summarize the current landscape, updates and future perspectives of immunotherapy in HCC.

\section{RATIONALE FOR IMMUNOTHERAPY IN HCC}

HCC arises in a chronically inflamed background in the vast majority of cases. An underlying liver disease derived from a viral infection or from a non-infectious condition occurs in around $90 \%$ of HCC patients ${ }^{[14]}$. The immunologic composition of the liver is crucial for the role of this organ in the entero-hepatic circulation as it exerts an immunologic control function under physiological condition ${ }^{[15]}$.

Liver has an important function in host defense and self-tolerance by the coordinated activity of a diverse immune-cell repertoire. Liver sinusoidal endothelial cells regulates the effector immune response by inhibiting $\mathrm{CD} 4+$ and $\mathrm{CD} 8+\mathrm{T}$ lymphocytes, thus preventing an immune reaction against bacterial antigens coming from the gut. Moreover, these cells express high levels of Program death receptor ligand 1 (PD-L1), which is a transmembrane immunosuppressive protein that inactivates the adaptative immune system by binding to the inhibitory lymphocyte receptor $\mathrm{PD}-1^{[16]}$. 
Kupffer cells, which are stationary macrophages in the liver sinusoids, contribute to immune tolerance by producing inhibitory cytokines such as IL-10 and prostaglandins and by expanding inhibitory regulatory $\mathrm{T}$ cells. Kupfer cells also plays a major role in the clearance of gut-derived endotoxins from the portal circulation ${ }^{[17]}$.

Tolerance-inducing cells, such as inhibitory $\mathrm{CD} 4+\mathrm{CD} 25+\mathrm{FoxP} 3+\mathrm{T}$ regulatory (Treg) cells, are increased in tumor tissue and peripheral blood of HCC patients ${ }^{[18]}$. Tregs can impar the effector function of intratumoral CD8+ T cells by several mechanisms, such as IL-10 and transforming growth factor (TGF) beta $\operatorname{production}^{[19]}$.

Neutrophils have been shown to induce tumor cell proliferation and stimulate angiogenesis through the secretion of cytokines ${ }^{[20]}$. Infiltrating neutrophils have been shown to recruit Tregs in animal HCC models and the number of neutrophils in HCC infiltrate is reported to be a negative prognostic factor in HCC patients submitted to resection ${ }^{[21]}$.

Myeloid-derived suppressor cells (MDSCs) are myeloid progenitor cells that acts as suppressor of Natural Killer (NK) cells and T cell effector function in the tumor microenvironment mediated by the expression of arginase, that depletes arginine, which is essential for $\mathrm{T}$ cell proliferation and also by the release of reactive oxygen species ${ }^{[22,23]}$. The MDSC subset has been reported as a prognostic factor for HCC recurrence after local treatment ${ }^{[24]}$.

Besides the activity of these immune cells, the liver micro-environment overexpresses immune checkpoint molecules, which can downregulate immune responses against tumor cells. PD-1/PD-L1 expression is observed not only in Kupffer cells, but also in tumor infiltrating lymphocytes. An association between PD-1 expression by T CD8+ lymphocytes and poor prognosis in HCC patients is reported ${ }^{[25]}$. T-cell immunoglobulin and mucin-domain-containing molecule- 3 is expressed by cells from innate and adaptive immune system and interacts with several ligands such as Galectin 3, which is expressed in liver tissue. Evidence indicates that Galectin-9 inhibits T-cell responses, acting as an immunosuppressive factor. Lymphocyte-activation gene 3 (LAG3) is a membrane protein that is expressed in activated T-cells and suppresses dendritic cells function. LAG3 acts symmetrically with PD-1 to promote cancer evasion from immune recognition ${ }^{[26]}$.

The hepatic chemokine profile also plays a substantial role in modulating immune response. It has been demonstrated that immunosuppressive cytokines such as IL-4, IL-5 and IL-10 are upregulated, while some pro-inflammatory cytokines such as TNF and IL-1 are downregulated, what can facilitate HCC $\operatorname{progression}^{[27]}$.

Tumor mutational burden is a measurement of mutations carried by tumor cells that seems to be correlated with cytotoxic T cells infiltration and better response to immune-checkpoint inhibitors (ICI) ${ }^{[28,29]}$. The mutational burden typically translates into a higher neo-antigen load, and therefore a higher chance that an antigen capable of stimulating an immune reaction is expressed on the tumor cell surface. Nevertheless, HCC ranks only as a medium mutated tumor, with an average of 5 somatic mutations per megabase, corresponding to approximately 60 non-synonymous substitutions within expressed genes. This accounts for a likely lower neoantigen burden comparing to melanoma, for example ${ }^{[30]}$.

The current research activity on immunotherapy for HCC are mainly based on targeting the abovementioned mechanisms. The complexity of immune system and the dismal prognosis of HCC are barriers for the translation of basic research from bench-to-bedside. In this regard, the development of cancer vaccines, adoptive cellular therapies, ICI and combinations of immunotherapy with other agents or with different treatment modalities are being developed and tested in the clinical setting. 


\section{CANCER VACCINES}

The number of vaccine trials in HCC is limited and the results showed only a modest efficacy. The aim of cancer vaccines is to stimulate the immune cells to recognize and attack tumor cells by managing tumor antigens and maturated dendritic cells, which makes the connection between innate and adaptative immune system ${ }^{[31]}$.

In HCC, a restricted number of tumor-associated antigens has been identified. Research in HCC vaccines mainly focus on alpha-fetoprotein (AFP) because this is usually expressed in this malignancy ${ }^{[32]}$. Initially, a study was conducted based on an AFP-derived peptide and was able to produce T-cell responses. In another study, dendritic cells pulsed with a lysate of HepG2 cell line showed safety and evidence of activity in patients with advanced $\mathrm{HCC}^{[33]}$. El Ansary et al.$^{[34]}$ also observed modest clinical responses, increasing in $\mathrm{CD} 8+$ lymphocytes count and in serum interferon concentration using an autologous dendritic cell $\operatorname{vaccine}^{[34]}$.

The "Cancer Vaccine development for Hepatocellular Carcinoma" - HEPAVAC project has the goal of developing strategies for a therapeutic peptide-based vaccine for HCC including both "off-the-shelf" and personalized antigens. A multi-epitope vaccine (IMA970A) is currently under evaluation in early/ intermediate HCC patients in a phase I/II European multicenter clinical trial (NCT03203005). The actual study start date was September-2017 and the completion date is expected to be on January-2020.

Combination of dendritic cell infusion following trans-catheter hepatic arterial embolization was shown to be safe and to enhance tumor-specific responses more effectively than embolization alone, but recurrence was not completely prevented ${ }^{[35]}$.

The PHOCUS trial, aimed to compare an oncolytic vaccine virus armed with granulocyte-macrophage colony stimulating factor gene $(J X-594)$ to sorafenib halted patient enrollment following a planned interim futility analysis that conclude that the trial was unlike to meet its primary endpoint of overall survival in the final analysis ${ }^{[36]}$. This oncolytic vaccine virus is also in a phase I/II trial in combination with nivolumab in first-line treatment of advanced HCC (NCT03071094).

Up to now, no HCC vaccine is approved for clinical use. Further research on development and applicability of this strategy, together with the completion of the active trials is warranted.

\section{CELL-BASED IMMUNOTHERAPY}

Several approaches involving cell-based treatments are being evaluated in HCC. Briefly, this modality consists of the use of autologous effector cells that are manipulated, expanded and sensitized ex vivo before being delivered to the patient ${ }^{[37]}$. Different types of lymphocytes, engineered $\mathrm{T}$ cell receptor (TCR) and chimeric antigen receptor (CAR) composes the mainstay of cell-based immunotherapy.

In the late 1980s, Onishi et al ${ }^{[38]}$ innovatively reported clinical responses in HCC patients treated with a combination of IL-2 and lymphokine-activated killer (LAK) cells showing a favorable safety profile ${ }^{[38]}$. However, further studies with LAK cells showed conflicting results in terms of efficacy ${ }^{[39]}$.

The post-operative use of isolated and expanded tumor-infiltrating lymphocytes is a promising strategy in HCC. In a study with 12 patients submitted to surgery, this therapy was associated with decreased recurrence rates at 6 and 12 months after resection comparing to a control group ${ }^{[40]}$. Cytokine-induced killer (CIK) cells, which comprises a mixture of T lymphocytes (CD3+/CD56+ cells, CD3-/CD56+ NK cells and CD3+/CD56cytotoxic T cells) provided longer progression-free survival (PFS) after HCC resection in a randomized trial 
involving 150 patients, with an acceptable toxicity profile ${ }^{[41]}$. In another randomized trial, adjuvant CIK cells provided longer time to recurrence, but with no impact in overall survival $(\mathrm{OS})^{[41]}$. An open-label phase III trial tested the efficacy of CIK cells as an adjuvant therapy after resection or percutaneous treatment. The study included 230 patients with HCC to receive CIK cells or no adjuvant treatment. The study met its primary endpoint, with a significant improvement in recurrence-free survival and OS with activated CIK cells ${ }^{[42]}$.

NK cells are also being studied in the HCC field. An ongoing phase II trial (NCT02008929) is focused on safety and efficacy of ex vivo expanded allogeneic NK cells in patients with high risk of recurrence after surgical resection and a second phase II trial (NCT02854839) is evaluating the role of NK adoptive cells after trans-arterial chemoembolization (TACE).

One of the most promising cellular therapies consists in the use of CAR T cells, which combines adoptive cellular immunotherapy with targeted therapy throughout receptor proteins that have been engineered to give $\mathrm{T}$ cells ability to recognize a specific protein independent of MHC. This approach has shown positive results when targeting CD19 via CAR for B-cell malignancies ${ }^{[43]}$. Currently, there are active clinical trials with CAR T cells in HCC with different target proteins, such as Glypican 3 (NCT02905188; NCT03146234 and NCT02723942). Nevertheless, a phase I/II trial with CAR-T anti-VEGFR2 (NCTo1218867) in metastatic tumors (with HCC patients allowed to be enrolled) failed to show a significant clinical activity in preliminary results.

Antigens from hepatitis virus B (HBV) can be potentially used as a target in adoptive cellular therapy, since HBV antigens can be found in both primary tumor and HCC metastasis. In a case report, HCC tumor cells were recognized in vivo by lymphocytes engineered to express an HBV-specific TCR. Therefore, this strategy has a potential to control HBV-associated $\mathrm{HCC}^{[44]}$. An AFP directed therapy is also being investigated in a phase I trial with autologous T cells with AFP-specific TCR in advanced HCC patients (NCT03132792).

Considering this scenario, adoptive cellular therapies for HCC showed an acceptable safety profile and seems to be an encouraging strategy in the adjuvant setting. However, confirmatory studies are required and there is no sufficient evidence to support its use in clinical practice outside clinical trials.

\section{IMMUNE CHECKPOINT INHIBITORS}

A major boost in the field of immunotherapy for cancer treatment came with the advent of immune checkpoint inhibitors (ICI). This class of drugs provided crucial improvements in the management of several tumors, such as lung cancer ${ }^{[45]}$, melanoma ${ }^{[46]}$ and others. The ICIs currently approved for clinical use target either CTLA-4 or PD/PD-L1 pathways. Tremelimumab, which is an anti-CTLA4 monoclonal antibody, was the first ICI evaluated in a clinical trial involving HCC patients. In a phase II study, 21 patients with chronic hepatitis $\mathrm{C}$ virus ( $\mathrm{HCV}$ ) infection not eligible for surgery received tremelimumab $15 \mathrm{mg} / \mathrm{kg}$ every 90 days for a maximum of 4 doses. A disease control rate of $76.4 \%$ was achieved, with $45 \%$ of these responses lasting more than 6 months. Few patients presented grade $3 / 4$ adverse events and tremelimumab was considered well tolerated. Besides, a significant drop in HCV viral load was observed among patients enrolled in this trial ${ }^{[47]}$.

Tremelimumab was also tested in a trial combining tumor ablation or TACE considering the hypothesis that tumor destruction by local therapies could enhance antigenic release and stimulate a systemic immune response. Thirty-two patients received tremelimumab every 4 weeks at two dosages ( 3.5 and $10 \mathrm{mg} / \mathrm{kg}$ ) for a total of 6 infusions, followed by an infusion every 3 months. The local therapy consisted of TACE in patients 
A

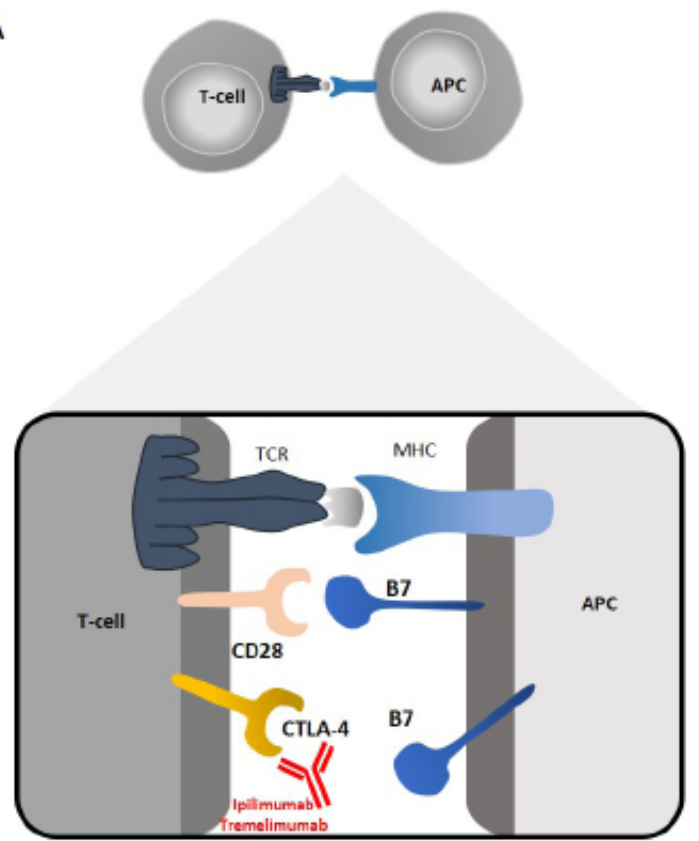

APC: antigen-presenting cell; TCR: T cell receptor; $M H C$ : major histocompatibility complex; CTLA: Cytotoxic Tlymphocyte antigen.
B
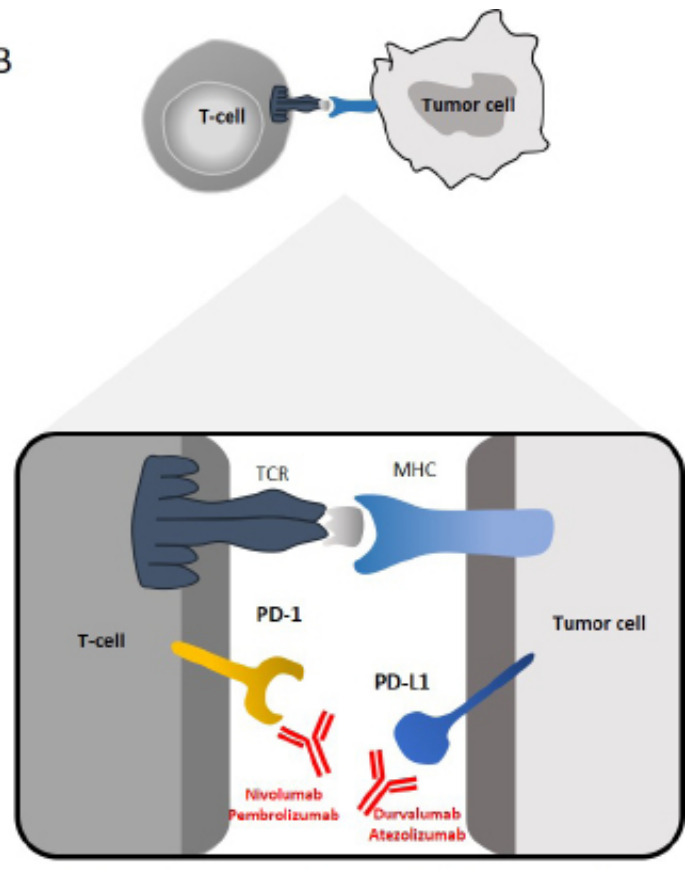

TCR: T cell receptor; MHC: major histocompatibility complex; PD: programmed cell death; L: ligand

Figure 1. (A) Mechanism of action for ipilimumab and tremelimumab. Cytotoxic T-lymphocyte antigen-4 (CTLA-4) is a negative regulator of T-cell activity. T-cell activation requires two separate stimulatory signals. The first signal occurs when the TCR binds to the major histocompatibility complex (MHC) of an antigen-presenting cell (APC). The second signal, or co-stimulatory signal, occurs when the CD28 receptor of T cells binds the B7 ligand of APCs. CTLA-4 is a naturally occurring T-cell receptor that, when bound by B7 on APCs, prevents the co-stimulation required for T-cell activation and suppresses T-cell activity. Ipilimumab and tremelimumab are monoclonal antibody designed to bind CTLA-4 and prevent its binding of B7, allowing for T-cell activation and potentiation to occur, allowing for enhanced immune-mediated cytotoxicity. (B) Mechanism of action for immune checkpoint inhibitors: The binding of PDL-1 on tumor cells to PD-1 on T-cells prevents T cells from killing tumor cells. Blocking the binding of PD-L1 to PD-1 with an immune checkpoint inhibitor allows cytotoxic activity of T cells against tumor cells. TCR: T cell receptor; PD: programmed cell death; L: ligand

with intermediate stage and ablation in patients with advanced stage. Partial responses were observed in $26 \%$ and stable disease in $63 \%$, with a median time-to-progression of 7.4 months and a median OS of 12.3 months. Again, no signals of serious toxicities were observed. The most common clinical adverse events was pruritus (9\%) while increasing transaminases was the most common laboratorial alteration $(34 \%)^{[48]}$.

Considering the high expression of PD-L1 in HCC cells and also in liver microenvironment components, strategies aimed to the PD1/PD-L1 pathway are been extensively tested in clinical trials. The mechanism of action of ICI directed to PD-1/PD-L1 is showed in Figure 1.

In a small phase I/II trial, the anti-PD-L1 agent durvalumab was evaluated in 40 HCC patients, most of them had been previously treated with sorafenib. An overall response rate of $10 \%$ with $20 \%$ of grade 3 or 4 toxicities were reported No treatment-related deaths were registered in this trial ${ }^{[49]}$.

The first data with nivolumab in HCC were published in 2017. The CHECKMATE 040 trial was an open label phase I/II study that included patients with intermediate and advanced HCC, who had progressed on or were intolerant to sorafenib and with HBV, HCV or non-infectious etiology. The first part of this trial evaluated a dose escalation cohort of 48 patients who were given nivolumab in doses ranging from $0.3 \mathrm{mg} / \mathrm{kg}$ up to $10 \mathrm{mg} / \mathrm{kg}$ every 2 weeks. The dose of $3 \mathrm{mg} / \mathrm{kg}$ was chosen for the further step, which consisted of a dose expansion cohort involving 214 patients aimed to test efficacy and safety. Toxicities 
did not vary across the different etiologies and the most common events were rash (23\%), pruritus (21\%) and diarrhea (13\%). Less than $2 \%$ presented serious adverse events of grade 3 or higher and no treatmentrelated death was observed. The response rate by RECIST 1.1 was $15 \%$ and $20 \%$ in the dose escalation and expansion cohorts, respectively, with a median duration of response in the expansion cohort of 9.9 months. The median OS was 28.6 months in the population naïve to sorafenib and 15.6 months in the sorafenibexperienced $^{[50]}$. Based on these results, nivolumab was granted accelerated approval by the US Food and Drug Administration (FDA) in 2017 for patients previously treated with sorafenib ${ }^{[51]}$. However, the phase III trial CHECKMATE 459, which compared nivolumab to sorafenib in the first line was announced to be negative by the company responsible for nivolumab development ${ }^{[52]}$. The results of this trial were presented in the European Society for Medical Oncology 2019 congress: the median OS was 16.4 months for nivolumab and 14.7 months for sorafenib (Hazard ratio 0.85, 95\% confidence interval 0.72-1.02, $P=0.0752$ ), with a response rate of $15 \%$ for nivolumab and $7 \%$ for sorafenib ${ }^{[53]}$. Complete results are still pending to be published.

Pembrolizumab, a monoclonal antibody that also targets PD-1, was shown to be active and safe in a phase II trial. The KEYNOTE 224 enrolled 104 HCC patients with intolerance or progression after sorafenib to receive pembrolizumab $200 \mathrm{mg}$ every 3 weeks in a single arm design. The authors recorded a response rate of $17 \%$ and $33 \%$ of the patients had stable disease. Grade 3 treatment-related adverse events were reported in $24 \%$ of the patients, being that the most common were increased transaminasemia and fatigue ${ }^{[54]}$. These results substantiated the accelerated approval of pembrolizumab by the FDA in $2018^{[55]}$.

The KEYNOTE 240 trial, which was a phase III trial comparing pembrolizumab to placebo in the secondline, were recently presented. The study included 413 patients who were randomized and analyzed for a co-primary endpoint of PFS and OS. The median OS of the pembrolizumab arm was 13.9 months vs. 10.6 months for the placebo arm, what did not reach the pre-specified efficacy boundaries for statistical significance ${ }^{[56]}$. Therefore, the trial was not able to confirm the superiority of pembrolizumab over placebo, even though the safety profile was manageable and the clinical difference between the two arms in terms of median OS warrants a further exploration of the role of ICI in HCC.

ICIs combination with ipilimumab and nivolumab in patients with advanced stage disease previously treated with sorafenib yielded a response rate of $31 \%$, including $5 \%$ of complete responses, with a median duration of response of 17.5 months. The study randomized patients into 3 arms: nivolumab $1 \mathrm{mg} / \mathrm{kg}$ and ipilimumab $3 \mathrm{mg} / \mathrm{kg}$ every 3 weeks for 4 cycles followed by nivolumab $240 \mathrm{mg}$ every 2 weeks, nivolumab $3 \mathrm{mg} / \mathrm{kg}$ and ipilimumab $1 \mathrm{mg} / \mathrm{kg}$ every 3 weeks for four cycles followed by nivolumab $240 \mathrm{mg}$ every 2 weeks or nivolumab $3 \mathrm{mg} / \mathrm{kg}$ and ipilimumab $1 \mathrm{mg} / \mathrm{kg}$ every 6 weeks. The first arm experienced a median overall survival of 22.8 months, the second and third arms reached 12 and 13 months respectively. Overall, the combination was well tolerated, with $37 \%$ of all patients experiencing grades 3-4 treatment-related adverse events ${ }^{[57]}$. Tremelimumab plus durvalumab was associated with $20 \%$ of grade $3-4$ toxicities with no unexpected safety signals in an analysis from an a phase I/II trial with 40 patients who progressed on or were intolerant to sorafenib ${ }^{[58]}$. An ongoing phase III trial (NCT 03298451) is actually recruiting patients in the first-line setting to sorafenib $v s$. durvalumab $v s$. duralumab plus tremelimumab. Trials on immunotherapy are listed in Table 1.

A focus is being placed on the potential benefit of ICI as an adjuvant treatment after resection or ablation. In this sense, patients with high risk of recurrence are being enrolled in a phase III placebo-controlled trial with nivolumab in the adjuvant setting. The primary endpoint of this study is recurrence-free survival (NCT03383458). Similarly, pembrolizumab (NCT03867084) and durvalumab with or without bevacizumab (NCT03847428) are also been tested in the adjuvant setting in other phase III trials in patients who achieved complete response after resection or ablation. 
Table 1. Ongoing trials involving immune checkpoint inhibitors in hepatocellular carcinoma

\begin{tabular}{|c|c|c|c|c|c|}
\hline Drug & Identifier & Phase & $n$ & Setting & Current status \\
\hline \multicolumn{6}{|l|}{ Monotherapy } \\
\hline Nivolumab & NCT01658878 & $|/ I|$ & 42 & $1 \mathrm{~L} / 2 \mathrm{~L}$ & Completed \\
\hline Nivolumab & NCT01658878 & $\mathrm{I} / \mathrm{II}$ & 214 & $1 \mathrm{~L} / 2 \mathrm{~L}$ & Completed \\
\hline Nivolumab & NCT01658878 & $|/| I$ & 200 & $1 \mathrm{~L}$ & Completed \\
\hline Nivolumab & NCT01658878 & $\mathrm{I} / \mathrm{II}$ & 262 & $1 \mathrm{~L} / 2 \mathrm{~L}$ & Completed \\
\hline Nivolumab & NCT02576509 & III & 726 & $1 \mathrm{~L}$ & Recruiting \\
\hline Nivolumab & NCT03383458 & III & 520 & Adjuvant & Recruiting \\
\hline Pembrolizumab & NCT02702414 & $\|$ & 100 & $2 \mathrm{~L}$ & Completed \\
\hline Pembrolizumab & NCT02702401 & III & 408 & $2 \mathrm{~L}$ & Recruiting \\
\hline Pembrolizumab & NCT03062358 & III & 330 & $2 \mathrm{~L}$ & Recruiting \\
\hline Pembrolizumab & NCT03211416 & $|-| \mid$ & 27 & $1 \mathrm{~L}$ & Recruiting \\
\hline Pembrolizumab & NCT03867084 & III & 950 & Adjuvant & Recruiting \\
\hline Relatlimab & NCT01968109 & $|-| \mid$ & 168 & $2 \mathrm{~L}$ & Recruiting \\
\hline LY3321367/LY3300054 & NCT03099109 & । & 196 & $2 \mathrm{~L}$ & Recruiting \\
\hline BGB-A317 & NCT03412773 & III & 660 & $1 \mathrm{~L}$ & Recruiting \\
\hline SHR-1210 & NCT02989922 & $\|$ & 220 & $2 \mathrm{~L}$ & Completed \\
\hline REGN3767 & NCT03005782 & I & 546 & $2 \mathrm{~L}$ & Recruiting \\
\hline \multicolumn{6}{|l|}{ Combination } \\
\hline Nivolumab/Ipilimumab & NCT01658878 & ॥ & 620 & $2 \mathrm{~L}$ & Completed \\
\hline Nivolumab/Ipilimumab & NCT03222076 & $\|$ & 45 & Neoadjuvant & Recruiting \\
\hline Nivolumab/Ipilimumab & NCT03510871 & ॥ & 40 & Neoadjuvant & Recruiting \\
\hline Nivolumab/Pexavec & NCT03071094 & $\|$ & 30 & $2 \mathrm{~L}$ & Recruiting \\
\hline Durvalumab/Tremelimumab & NCT02519348 & II & 545 & $1 \mathrm{~L} / 2 \mathrm{~L}$ & Recruiting \\
\hline Durvalumab/Tremelimumab & NCT03298451 & III & 1200 & $1 \mathrm{~L}$ & Recruiting \\
\hline Relatlimab/Nivolumab & NCT01968109 & $|-| \mid$ & 168 & $2 \mathrm{~L}$ & Recruiting \\
\hline REGN3767/REGN2810 & NCT03005782 & I & 546 & $2 \mathrm{~L}$ & Recruiting \\
\hline LY3321367/LY3300054 & NCT03099109 & I & 196 & $2 \mathrm{~L}$ & Recruiting \\
\hline Atezolizumab/Bevacizumab & NCT03434379 & III & 480 & $1 \mathrm{~L}$ & Recruiting \\
\hline PDR001/FGF401 & NCT02325739 & $\|$ & 238 & $2 \mathrm{~L}$ & Recruiting \\
\hline PDR001/INC280 & NCT02795429 & $\|$ & 108 & $2 \mathrm{~L}$ & Recruiting \\
\hline Nivolumab/Galunisertib & NCT02423343 & ॥ & 75 & $2 \mathrm{~L}$ & Completed \\
\hline Regorafenib/Pembrolizumab & NCT03347292 & I & 40 & $1 \mathrm{~L}$ & Recruiting \\
\hline Cabozantinib/Nivolumab & NCT03299946 & I & 15 & Neoadjuvant & Recruiting \\
\hline Nivolumab/CC-122 & NCT02859324 & $|-| \mid$ & 50 & $2 \mathrm{~L}$ & Recruiting \\
\hline PDR001/Sorafenib & NCT02988440 & $\|$ & 50 & $2 \mathrm{~L}$ & Recruiting \\
\hline Pembrolizumab/Lenvatinib & NCT03006926 & । & 104 & $2 \mathrm{~L}$ & Recruiting \\
\hline Nivolumab/TACE & NCT03143270 & । & 14 & $2 \mathrm{~L}$ & Recruiting \\
\hline Nivolumab/Y90 & NCT03033446 & ॥ & 40 & $2 \mathrm{~L}$ & Recruiting \\
\hline
\end{tabular}

In the neoadjuvant setting, the study PRIME-HCC (NCT 03682276) is recruiting patients to assess safety and activity of nivolumab plus ipilimumab prior to liver resection in HCC in centers from United Kingdom.

\section{COMBINATION STRATEGIES}

Besides ongoing trials with immunotherapies, there has been active research on combination of immunotherapy with other treatments such as tyrosine kinase inhibitors, ablative therapies, anti-VEGF antibodies and combination of different ICI. Some of these modalities can potentially improve treatment outcomes due to synergistic effects caused by tumor cell death on immune response. T cell activation and pro-inflammatory cytokines release are described to occur few weeks after locoregional therapies for $\mathrm{HCC}^{[59]}$.

There are ongoing trials testing the combination of ICI with Ytrium-90 (NCT 03033446 and NCT 02837029), TACE (NCT 03143270) and after liver resection or ablation (NCT03847428). The dual 
combination of ICI (anti-CTLA-4 and PD1/PD-L1) is also being evaluated in clinical trials, for example, tremelimumab plus durvalumab (NCT 02119348) and ipilimumab plus nivolumab (NCT01658878).

Encouraging results of the combination of bevacizumab (an anti-VEGF antibody) with atezolizumab (an anti-PD-L1 ICI) were reported in a phase Ib study that included 68 HCC patients. It was reported a response rate of $34 \%$, with 19 of the 23 responses lasting longer than 6 months. Grade 3-4 adverse events were seen in $25 \%$ of the patients ${ }^{[60]}$. This combination was granted a breakthrough designation therapy by the US FDA in $2018^{[6]]}$ and a phase III trial aimed to evaluated atezolizumab and bevacizumab $v$ s. sorafenib is under recruitment (NCT0343479).

An open-label phase $1 \mathrm{~b}$ trial that assessed the efficacy of lenvatinib plus pembrolizumab in 94 patients has been recently reported. The combination induced a confirmed response rate of $26.9 \%$, with a median PFS of 9.69 months. Sixty percent of the patients had dose interruptions or reductions, 5 patients had serious adverse events and there were 2 treatment-related deaths ${ }^{[62]}$. Avelumab with axitinib also showed encouraging activity in a trial with 22 patients, although a higher rate of grade 3 hypertension $(50 \%)$ and hand-foot skin reaction $(22.7 \%)$ were reported ${ }^{[63]}$. Table 2 summarizes main results on ICI in HCC.

\section{CHALLENGES AND FUTURE DIRECTIONS}

While final results on immunotherapy for HCC are awaited, some relevant issues are to be taken into account when interpreting the available and upcoming data.

ICIs are associated with atypical patterns of response and progression. The traditional radiologic criteria used to evaluate tumor response in oncology trials with cytotoxic chemotherapy may not be accurate enough to detect clinical benefit or treatment failure with immune oncology agents. For example, a radiological increasing in tumor burden without worsening in disease burden, called pseudoprogression, can be explained by an immune-cell infiltration and do not represent treatment failure ${ }^{[64]}$. Patients with an initial progressive disease followed by a later radiologic response may experience a nonconventional survival benefit comparing to those patients with the same initial behavior ${ }^{[65]}$. Therefore, treatment with ICI warrants specific radiologic criteria to assess benefit or the emergence of resistance.

Around $10 \%-30 \%$ of the patients with other solid tumors submitted to ICI treatment present long-term disease control. This finding suggests the existence of a subgroup of patients that probably presents a sensitive tumoral phenotype or a specific predictive biomarker. Mutational burden, tumor-infiltrating lymphocytes and immune gene signatures are also being investigated as potential tools ${ }^{[66]}$. For HCC, the PDL-1 expression seems to be around 20\%-25\%, but no correlation between PDL-1 expression and better response has been established so far ${ }^{[67]}$.

Immune-related adverse events induced by ICIs are also a major concern in HCC. A wide range of events are described in immunotherapy trials (dermatologic, endocrinologic, gastrointestinal and others), but hepatotoxicity is of particular interest in this context. In clinical trials with anti-PD1 inhibitors, liver enzymes elevations were typically mild. However, HCC patients often present underlying cirrhosis with a limited liver reserve, what increase the risk of decompensation even in mild liver alterations.

In conclusion, the incorporation of immunotherapy in the HCC landscape is still under development. The recently announced negative results of the phase III trials with pembrolizumab ${ }^{[56]}$ and nivolumab ${ }^{[52]}$ somehow disappointed the initial hope placed in this strategy. Immunotherapy for HCC seems to be a tougher road comparing to what we are experiencing with other tumors. However, huge effort is being made in the search for predictive biomarkers and development of novel strategies to deliver better outcomes for HCC patients. 


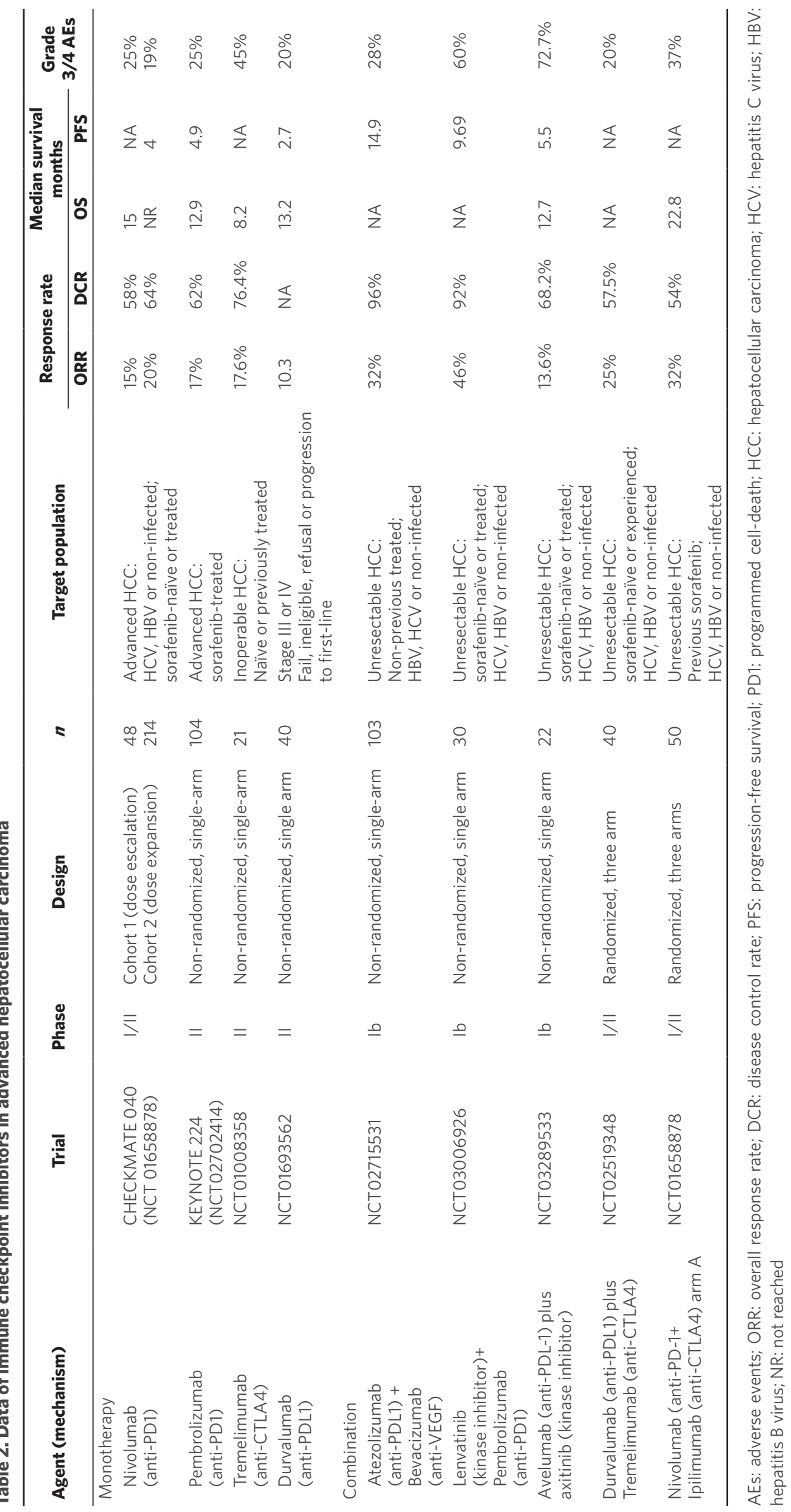




\section{DECLARATIONS}

\section{Authors' contributions}

Discussion, writing and editing of the manuscript: da Fonseca LG

Discussion, writing and editing of the manuscript: Carrilho FJ

\section{Availability of data and materials}

Not applicable.

\section{Financial support and sponsorship}

None.

\section{Conflicts of interest}

Both authors declared that there are no conflicts of interest.

\section{Ethical approval and consent to participate}

Not applicable.

\section{Consent for publication}

Not applicable.

\section{Copyright}

(C) The Author(s) 2019.

\section{REFERENCES}

1. IARC. Fact sheets by Population-Globocan-IARC. Available from: https://gco.iarc.fr/today/data/factsheets/cancers/39-All-cancersfact-sheet.pdf

2. Ishizawa T, Hasegawa K, Aoki T, Takahashi M, Inoue Y, et al. Neither multiple tumors nor portal hypertension are surgical contraindications for hepatocellular carcinoma. Gastroenterology 2008;134:1908-16.

3. Llovet JM, Ricci S, Mazzaferro V, Hilgard P, Gane E, et al. Sorafenib in advanced hepatocellular carcinoma. N Engl J Med 2008;359:378-90.

4. Cheng AL, Kang YK, Chen Z, Tsao CJ, Qin S, et al. Efficacy and safety of sorafenib in patients in the Asia-Pacific region with advanced hepatocellular carcinoma: a phase III randomised, double-blind, placebo-controlled trial. Lancet Oncol 2009;10:25-34.

5. Kudo M, Finn RS, Qin S, Han KH, Ikeda K, et al. Lenvatinib versus sorafenib in first-line treatment of patients with unresectable hepatocellular carcinoma: a randomised phase 3 non-inferiority trial. Lancet 2018;391:1163-73.

6. Bruix J, Qin S, Merle P, Granito A, Huang YH, et al. Regorafenib for patients with hepatocellular carcinoma who progressed on sorafenib treatment (RESORCE): a randomised, double-blind, placebo-controlled, phase 3 trial. Lancet 2017;389:56-66.

7. Abou-Alfa GK, Meyer T, Cheng AL, El-Khoueiry AB, Rimassa L, et al. Cabozantinib in patients with advanced and progressing hepatocellular carcinoma. N Engl J Med 2018;379:54-63.

8. Zhu AX, Kang YK, Yen CJ, Finn RS, Galle PR, et al. Ramucirumab after sorafenib in patients with advanced hepatocellular carcinoma and increased $\alpha$-fetoprotein concentrations (REACH-2): a randomised, double-blind, placebo-controlled, phase 3 trial. Lancet Oncol 2019;20:282-96.

9. Finn RS, Merle P, Granito A, Huang YH, Bodoky G, et al. Outcomes of sequential treatment with sorafenib followed by regorafenib for HCC: additional analyses from the phase III RESORCE trial. J Hepatol 2018;69:353-8.

10. Coley WB. The treatment of malignant tumors by repeated inoculations of erysipelas. With a report of ten original cases. Am J Med Sci 1893;105:487-510.

11. Dunn GP, Bruce AT, Ikeda H, Old LJ, Schreiber RD. Cancer immunoediting: from immunosurveillance to tumor escape. Nat Immunol 2002;3:991-8.

12. Morales A, Eidinger D, Bruce AW. Intracavitary Bacillus Calmette-guerin in the treatment of superficial bladder tumors. J Urol 1976;116):180-2.

13. Rudnicka D, Oszmiana A, Finch DK, Strickland I, Schofield DJ, et al. Rituximab causes a polarization of B cells that augments its therapeutic function in NK-cell-mediated antibody-dependent cellular cytotoxicity. Blood 2013;121:4694-702.

14. Forner A, Reig M, Bruix J. Hepatocellular carcinoma. Lancet 2018;391:1301-14.

15. Pardee AD, Butterfield LH. Immunotherapy of hepatocellular carcinoma: Unique challenges and clinical opportunities. Oncoimmunology 2012;1:48-55. 
16. Shetty S, Lalor PF, Adams DH. Liver sinusoidal endothelial cells - gatekeepers of hepatic immunity. Nat Rev Gastroenterol Hepatol 2018;15:555-67.

17. Dixon LJ, Barnes M, Tang H, Pritchard MT, Nagy LE. Kupffer cells in the liver. Compr Physiol 2013;3:785-97.

18. Fu J, Xu D, Liu Z, Shi M, Zhao P, et al. Increased regulatory T cells correlate with CD8 T-cell impairment and poor survival in hepatocellular carcinoma patients. Gastroenterology 2007;132:2328-39.

19. Sprinzl MF, Galle PR. Immune control in hepatocellular carcinoma development and progression: role of stromal cells. Semin Liver Dis 2014;34:376-88

20. Mollinedo F. Neutrophil degranulation, plasticity, and cancer metastasis. Trends Immunol 2019;40:228-42.

21. Zhou SL, Zhou ZJ, Hu ZQ, Huang XW, Wang Z, et al. Tumor-associated neutrophils recruit macrophages and T-regulatory cells to promote progression of hepatocellular carcinoma and resistance to Sorafenib. Gastroenterology 2016;150:1646-58.e17.

22. Draghiciu O, Lubbers J, Nijman HW, Daemen T. Myeloid derived suppressor cells-An overview of combat strategies to increase immunotherapy efficacy. Oncoimmunology 2015;4:e954829.

23. Kumar V, Patel S, Tcyganov E, Gabrilovich DI. The nature of myeloid-derived suppressor cells in the tumor microenvironment. Trends Immunol 2016;37:208-20.

24. Mizukoshi E, Yamashita T, Arai K, Terashima T, Kitahara M, et al. Myeloid-derived suppressor cells correlate with patient outcomes in hepatic arterial infusion chemotherapy for hepatocellular carcinoma. Cancer Immunol Immunother 2016;65:715-25.

25. Calderaro J, Rousseau B, Amaddeo G, Mercey M, Charpy C, et al. Programmed death ligand 1 expression in hepatocellular carcinoma: Relationship With clinical and pathological features. Hepatology 2016;64:2038-46.

26. Yan W, Liu X, Ma H, Zhang H, Song X, et al. Tim-3 fosters HCC development by enhancing TGF- $\beta$-mediated alternative activation of macrophages. Gut 2015;64:1593-604.

27. Budhu A, Forgues M, Ye QH, Jia HL, He P, et al. Prediction of venous metastases, recurrence, and prognosis in hepatocellular carcinoma based on a unique immune response signature of the liver microenvironment. Cancer Cell 2006;10:99-111.

28. Brown SD, Warren RL, Gibb EA, Martin SD, Spinelli JJ, et al. Neo-antigens predicted by tumor genome meta-analysis correlate with increased patient survival. Genome Res 2014;24:743-50.

29. Schumacher TN, Schreiber RD. Neoantigens in cancer immunotherapy. Science 2015;348:69-74.

30. Fujimoto A, Furuta M, Totoki Y, Tsunoda T, Kato M, et al. Whole-genome mutational landscape and characterization of noncoding and structural mutations in liver cancer. Nat Genet 2016;48:500-9.

31. Lee HJ, Kim SK, Cho D, Lee JJ. Cellular immunotherapy as a beacon of hope for hematological malignancies. Blood Res 2015;50:126-8.

32. Schmidt N, Neumann-Haefelin C, Thimme R. Cellular immune responses to hepatocellular carcinoma: lessons for immunotherapy. Dig Dis 2012;30:483-91.

33. Palmer DH, Midgley RS, Mirza N, Torr EE, Ahmed F, et al. A phase II study of adoptive immunotherapy using dendritic cells pulsed with tumor lysate in patients with hepatocellular carcinoma. Hepatology 2009;49:124-32.

34. El Ansary M, Mogawer S, Elhamid SA, Alwakil S, Aboelkasem F, et al. Immunotherapy by autologous dendritic cell vaccine in patients with advanced HCC. J Cancer Res Clin Oncol 2013;139:39-48.

35. Mizukoshi E, Nakamoto Y, Arai K, Yamashita T, Mukaida N, et al. Enhancement of tumor-specific T-cell responses by transcatheter arterial embolization with dendritic cell infusion for hepatocellular carcinoma. Int J cancer 2010;126:2164-74.

36. Pexa-Vec/Nexavar combination fails phase III trial in liver cancer. Available from: https://www.genengnews.com/news/pexa-vecnexavar-combination-fails-phase-iii-trial-in-liver-cancer/. [Last accessed on 9 Oct 2019]

37. Liu D, Staveley-O'Carroll KF, Li G. Immune-based therapy clinical trials in hepatocellular carcinoma. J Clin Cell Immunol 2015;6:376.

38. Onishi S, Saibara T, Fujikawa M, Sakaeda H, Matsuura Y, et al. Adoptive immunotherapy with lymphokine-activated killer cells plus recombinant interleukin 2 in patients with unresectable hepatocellular carcinoma. Hepatology 1989;10:349-53.

39. Kawata A, Une Y, Hosokawa M, Wakizaka Y, Namieno T, et al. Adjuvant chemoimmunotherapy for hepatocellular carcinoma patients. Adriamycin, interleukin-2, and lymphokine-activated killer cells versus adriamycin alone. Am J Clin Oncol 1995;18:257-62.

40. Wang Y, Chen H, Wu M, Bao J, Cong W, et al. Postoperative immunotherapy for patients with hepatocarcinoma using tumorinfiltrating lymphocytes. Chin Med J (Engl) 1997;110:114-7.

41. Takayama T, Sekine T, Makuuchi M, Yamasaki S, Kosuge T, et al. Adoptive immunotherapy to lower postsurgical recurrence rates of hepatocellular carcinoma: a randomised trial. Lancet 2000;356:802-7.

42. Lee JH, Lee JH, Lim YS, Yeon JE, Song TJ, et al. Adjuvant immunotherapy with autologous cytokine-induced killer cells for hepatocellular carcinoma. Gastroenterology 2015;148:1383-91.e6.

43. Maus M V, Grupp SA, Porter DL, June CH. Antibody-modified T cells: CARs take the front seat for hematologic malignancies. Blood 2014;123:2625-35.

44. Qasim W, Brunetto M, Gehring AJ, Xue SA, Schurich A, et al. Immunotherapy of HCC metastases with autologous T cell receptor redirected T cells, targeting HBsAg in a liver transplant patient. J Hepatol 2015;62:486-91.

45. Reck M, Rodríguez-Abreu D, Robinson AG, Hui R, Csőszi T, et al. Pembrolizumab versus chemotherapy for PD-L1-positive nonsmall-cell lung cancer. N Engl J Med 2016;375:1823-33.

46. Robert C, Long GV, Brady B, Dutriaux C, Maio M, et al. Nivolumab in previously untreated melanoma without BRAF mutation. N Engl J Med 2015;372:320-30.

47. Sangro B, Gomez-Martin C, de la Mata M, Iñarrairaegui M, Garralda E, et al. A clinical trial of CTLA-4 blockade with tremelimumab in patients with hepatocellular carcinoma and chronic hepatitis C. J Hepatol 2013;59:81-8. 
48. Duffy AG, Ulahannan SV, Makorova-Rusher O, Rahma O, Wedemeyer H, et al. Tremelimumab in combination with ablation in patients with advanced hepatocellular carcinoma. J Hepatol 2017;66:545-51.

49. Wainberg ZA, Segal NH, Jaeger D, Lee KH, Marshall J, et al. Safety and clinical activity of durvalumab monotherapy in patients with hepatocellular carcinoma (HCC). J Clin Oncol 2017;35:4071.

50. El-Khoueiry AB, Sangro B, Yau T, Crocenzi TS, Kudo M, et al. Nivolumab in patients with advanced hepatocellular carcinoma (CheckMate 040): an open-label, non-comparative, phase 1/2 dose escalation and expansion trial. Lancet 2017;389:2492-502.

51. FDA grants accelerated approval to nivolumab for HCC previously treated with sorafenib. Available from: https://www.fda.gov/drugs/ informationondrugs/approveddrugs/ucm577166.htm. [Last accessed on 10 Oct 2019]

52. Bristol-Myers Squibb Announces Results from CheckMate -459 Study Evaluating Opdivo (nivolumab) as a First-Line Treatment for Patients with Unresectable Hepatocellular Carcinoma. Available from: https://news.bms.com/press-release/bmy/bristol-myers-squibbannounces-results-checkmate-459-study-evaluating-opdivo-nivol. [Last accessed on 10 Oct 2019]

53. Yau T, Park JW, Finn RS, Cheng AL, Mathurin P, et al. CheckMate 459: A randomized, multi-center phase III study of nivolumab (NIVO) vs sorafenib (SOR) as first-line (1L) treatment in patients (pts) with advanced hepatocellular carcinoma (aHCC). Ann Oncol 2019;30:mdz394.029

54. Zhu AX, Finn RS, Edeline J, Cattan S, Ogasawara S, et al. Pembrolizumab in patients with advanced hepatocellular carcinoma previously treated with sorafenib (KEYNOTE-224): a non-randomised, open-label phase 2 trial. Lancet Oncol 2018;19:940-52.

55. FDA grants accelerated approval to pembrolizumab for hepatocellular carcinoma. Available from: https://www.fda.gov/Drugs/ InformationOnDrugs/ApprovedDrugs/ucm625705.htm. [Last accessed on 10 Oct 2019]

56. Finn RS, Ryoo BY, Merle P, Kudo M, Bouattour M, et al. Results of KEYNOTE-240: phase 3 study of pembrolizumab (Pembro) vs best supportive care (BSC) for second line therapy in advanced hepatocellular carcinoma (HCC). J Clin Oncol 2019;37:abstract4004.

57. Yau T, Kang YK, Kim TY, El-Khoueiry AB, Santoro A, et al. Nivolumab (NIVO) + ipilimumab (IPI) combination therapy in patients (pts) with advanced hepatocellular carcinoma (aHCC): results from CheckMate 040. J Clin Oncol 2019;37:4012.

58. Kelley RK, Abou-Alfa GK, Bendell JC, Kim TY, Borad MJ, et al. Phase I/II study of durvalumab and tremelimumab in patients with unresectable hepatocellular carcinoma (HCC): phase I safety and efficacy analyses. J Clin Oncol 2017;35:4073.

59. Zhou Y, Xu X, Ding J, Jing X, Wang F, et al. Dynamic changes of T-cell subsets and their relation with tumor recurrence after microwave ablation in patients with hepatocellular carcinoma. J Cancer Res Ther 2018;14:40-5.

60. Pishvaian MJ, Lee MS, Ryoo B, Stein S, Lee K, et al. Updated safety and clinical activity results from a phase Ib study of atezolizumab + bevacizumab in hepatocellular carcinoma (HCC). Available from: https://oncologypro.esmo.org/Meeting-Resources/ ESMO-2018-Congress/Updated-safety-and-clinical-activity-results-from-a-Phase-Ib-study-of-atezolizumab-bevacizumab-inhepatocellular-carcinoma-HCC. [Last accessed on 10 Oct 2019]

61. FDA grants Breakthrough Therapy Designation for Roche's Tecentriq in combination with Avastin as first-line treatment for advanced or metastatic hepatocellular carcinoma (HCC). Available from: https://www.roche.com/media/releases/med-cor-2018-07-18.htm. [Last accessed on 10 Oct 2019]

62. Ikeda M, Sung MW, Kudo M, Kobayashi M, Baron AD, et al. A phase $1 \mathrm{~b}$ trial of lenvatinib (LEN) plus pembrolizumab (PEM) in patients (pts) with unresectable hepatocellular carcinoma (uHCC). J Clin Oncol 2018;36:abstract4076.

63. Kudo M, Motomura K, Wada Y, Sakamoto Y, Kurosaki M. First-line avelumab + axitinib in patients with advanced hepatocellular carcinoma: results from a phase $1 \mathrm{~b}$ trial (VEGF Liver 100). J Clin Oncol 2019;37:abstract4072.

64. Stein S, Pishvaian MJ, Lee MS, Lee KH, Hernandez S, et al. Safety and clinical activity of $1 \mathrm{~L}$ atezolizumab + bevacizumab in a phase Ib study in hepatocellular carcinoma (HCC). J Clin Oncol 2018;36:4074.

65. El-Khoueiry AB, Melero I, Yau TC, Crocenzi TS, Kudo M, et al. Impact of antitumor activity on survival outcomes, and nonconventional benefit, with nivolumab (NIVO) in patients with advanced hepatocellular carcinoma (aHCC): Subanalyses of CheckMate-040. J Clin Oncol 2018;36:475.

66. Gibney GT, Weiner LM, Atkins MB. Predictive biomarkers for checkpoint inhibitor-based immunotherapy. Lancet Oncol 2016;17:e542-51.

67. El-Khoueiry AB, Sangro B, Yau T, Crocenzi TS, Kudo M, et al. Nivolumab in patients with advanced hepatocellular carcinoma (CheckMate 040): an open-label, non-comparative, phase 1/2 dose escalation and expansion trial. Lancet 2017;389:2492-502. 